\title{
A Flat Triangular Form for Nonlinear Systems with Two Inputs: Necessary and Sufficient Conditions ${ }^{\text {th }}$
}

\author{
H.B. Silveira ${ }^{\mathrm{a}, *}$, P.S. Pereira da Silva ${ }^{\mathrm{b}}$, P. Rouchon ${ }^{\mathrm{c}}$ \\ ${ }^{a}$ Departamento de Automação e Sistemas (DAS), Federal University of Santa Catarina \\ (UFSC), Florianópolis, Brazil \\ ${ }^{b}$ Escola Politécnica - PTC, University of São Paulo (USP), São Paulo, Brazil \\ ${ }^{c}$ Centre Automatique et Systèmes (CAS), Mines ParisTech, Paris, France
}

\begin{abstract}
The present work establishes necessary and sufficient conditions for a nonlinear system with two inputs to be described by a specific triangular form. Except for some regularity conditions, such triangular form is flat. This may lead to the discovery of new flat systems. The proof relies on well-known results for driftless systems with two controls (the chained form) and on geometric tools from exterior differential systems. The paper also illustrates the application of its results on an academic example and on a reduced order model of an induction motor.
\end{abstract}

Keywords:

Differential flatness, exterior differential systems, nonlinear systems, triangular forms

\section{Introduction}

Consider the control system with two inputs

$$
\dot{x}=f(x)+g_{1}(x) u_{1}+g_{2}(x) u_{2},
$$

\footnotetext{
"The first author was fully supported by CAPES and FUNPESQUISA/UFSC. The second author was partially supported by CNPq. The third author was partially supported by "Agence Nationale de la Recherche" (ANR), Projet Blanc EMAQS number ANR-2011BS01-017.

${ }^{*}$ Corresponding author.

Email addresses: hector.silveira@ufsc.br (H.B. Silveira), paulo@lac.usp.br (P.S. Pereira da Silva), pierre.rouchon@mines-paristech.fr (P. Rouchon)
} 
where $x \in U \subset \mathbb{R}^{n}$ is the state, $U$ is open, $n \geq 2, u=\left(u_{1}, u_{2}\right) \in \mathbb{R}^{2}$ is the control and $f, g_{1}, g_{2}: U \rightarrow \mathbb{R}^{n}$ are smooth (i.e. infinitely differentiable) mappings. Recall that (1) is driftless when $f=0$ and that $u=\alpha(x)+\beta(x) v$ is a regular static state feedback defined on an open set $V \subset U$ if the mappings $\alpha=\left(\alpha_{1}, \alpha_{2}\right): V \rightarrow \mathbb{R}^{2}$ and $\beta=\left(\beta_{i j}\right): V \rightarrow \mathbb{R}^{2 \times 2}$ are smooth and the matrix $\beta(x)=\left(\beta_{i j}(x)\right) \in \mathbb{R}^{2 \times 2}$ is invertible, for all $x \in V$, where $v=\left(v_{1}, v_{2}\right) \in \mathbb{R}^{2}$ is the new control. It was established in [1] necessary and sufficient conditions for (1) with $f=0$ to be (locally) described around a given $x_{0} \in U$ by the chained form

$$
\begin{aligned}
\dot{z}_{1} & =z_{2} v_{1}, \\
& \vdots \\
\dot{z}_{n-2} & =z_{n-1} v_{1}, \\
\dot{z}_{n-1} & =v_{2}, \\
\dot{z}_{n} & =v_{1},
\end{aligned}
$$

after a change of coordinates $z=\varphi(x)$ and a regular feedback $u=\beta v$ (see Theorem 1 in Section 3). In order to take into account the drift $f$ in (1), this work adds some geometric conditions (concerning exterior differential systems) to the ones of [1] and presents necessary and sufficient conditions for (11) (with or without drift) to be described around a given $x_{0} \in U$ by the triangular form

$$
\begin{aligned}
\dot{z}_{1} & =\phi_{1}\left(z_{1}, z_{2}, z_{n}\right)+z_{2} v_{1}, \\
\dot{z}_{2} & =\phi_{2}\left(z_{1}, z_{2}, z_{3}, z_{n}\right)+z_{3} v_{1}, \\
& \vdots \\
\dot{z}_{n-3} & =\phi_{n-3}\left(z_{1}, z_{2}, \ldots, z_{n-2}, z_{n}\right)+z_{n-2} v_{1}, \\
\dot{z}_{n-2} & =\phi_{n-2}\left(z_{1}, z_{2}, \ldots, z_{n}\right)+z_{n-1} v_{1}, \\
\dot{z}_{n-1} & =v_{2}, \\
\dot{z}_{n} & =v_{1},
\end{aligned}
$$

after a change of coordinates $z=\varphi(x)$ and a regular static state feedback $u=\alpha+\beta v$. Such result is the main contribution of this paper (see Theorem 3 in Section (4). Note that the control vector fields in (2) and (3) are exactly the same, and that the drift $f$ is taken into account by means of condition 2 ) of Theorem [3. Furthermore, [2] also considers (1) with $f=0$ and exhibits 
sufficient conditions for the chained form description (2) to hold generically, that is, on an open dense set (see Theorem 22). This work then establishes as an immediate corollary of the main result sufficient geometric conditions for (11) to be generically described by the triangular form (3) (see Corollary 1).

There are many motivations for choosing the specific triangular form (3). Firstly, (3) is (essentially) flat. This is discussed in detail in the subsequent paragraphs. Secondly, (3) may be seen as a generalization of the chained form (2) to systems with drift. Although there are many possible such generalizations, the conditions obtained in this paper with respect to the triangular form (3) may be regarded as generalizations of the ones of [1] and [2] for the chained form (21), since new conditions have been added to them in order to consider the drift $f$ in (11). Thirdly, the control literature has recently demonstrated interest in the triangular form (3) (see e.g. [3]). To the best of the authors' knowledge, the first work in the literature that considered and characterized the triangular form (3) was the thesis [4, Teorema 3.10, p. 51] of one of the authors. The present paper slightly improves the results of [4] and illustrates the constructive aspects of the results in the examples. Lastly, [5] provides necessary and sufficient conditions for a multi-input nonlinear system to be described, after a change of coordinates, by a flat triangular canonical form which differs from (3). In Example 1 in Section 4, one exhibits a nonlinear system of the form (11) which is transformed into (31) by means of the application of the results here established, but which fails to meet the referred conditions of [5].

It is well-known (and immediate to verify) that $y=\left(z_{1}, z_{n}\right)$ is a flatt output for the chained form (2) around the points in which $v_{1} \neq 0$. For the triangular form (3), straightforward computations using the implicit function theorem show that $y=\left(z_{1}, z_{n}\right)$ is a flat output around the points that satisfy $v_{1}+\partial \phi_{1} / \partial z_{2}(z) \neq 0, \ldots, v_{1}+\partial \phi_{n-2} / \partial z_{n-1}(z) \neq 0$. Hence, except for these regularity conditions, this paper establishes sufficient conditions for (11) to be flat, which therefore may lead to the discovery of new flat systems. The corresponding constructive procedure for describing (11) by the form (3), and hence constructing a flat output, is illustrated in Example 2 at the end of Section 4. It may be summarized as follows (see Remark 3 for further details). Assume that both conditions in Theorem 3 are met. First, one constructs a change of coordinates $z=\varphi(x)$ and $\beta(x)$ in the regular feedback $u=\beta v$ such

\footnotetext{
${ }^{1}$ For the concept of flatness, see e.g. [6], 7], [8].
} 
that the control vector fields $g_{1}, g_{2}$ in (11) are described by the chained form (2) (there are available methods for this in the literature, and the existence of such $z=\varphi(x)$ and $u=\beta v$ is ensured by condition 1) of Theorem 3). Then, condition 2) of Theorem 3 implies that after applying the regular feedback $u=\alpha+\beta v$ in (1), where $\alpha=\beta \bar{\alpha}$ and $\bar{\alpha}=-\left(\left\langle d z_{n}, f\right\rangle,\left\langle d z_{n-1}, f\right\rangle\right)$, one has that the closed-loop drift $f+\alpha_{1} g_{1}+\alpha_{2} g_{2}$ exhibits the triangular structure in (3) in the coordinates $z=\varphi(x)$. Hence, (11) has been transformed into (3). Lastly, $y=\left(z_{1}, z_{n}\right)$ is the resulting flat output constructed.

One recalls that the practical importance of flat systems is that the control problems of motion planning, trajectory tracking and flat output tracking are easily (and sometimes trivially) treated [6, 7, 8]. It is still an open problem in the control literature how to determine if a general nonlinear system is flat and, in such case, how a flat output can be constructed. As is well-known, a system which is linearizable by regular static state feedback is flat, but the converse is false. The results here presented could establish that a system of the form (11) is flat even when it is not linearizable by regular static feedback. This is the case, for instance, when the distribution determined by the control vector fields $g_{1}, g_{2}$ in (11) is not involutive, so that (11) is not static feedback linearizable (see e.g. [9, Proposition 9.16]). Nonetheless, such system could still meet the conditions in Theorem 3 (or Corollary 1) of the present work, in which case $y=\left(z_{1}, z_{n}\right)$ would be a flat output. Therefore, the results here established may lead to the discovery of new flat systems. As a final remark, it is clear that not all flat systems are static feedback equivalent to the triangular form (3). Indeed, if (1) is static feedback linearizable, then the distribution spanned by $g_{1}, g_{2}$ must be involutive, and hence condition 1) of Theorem 3) is violated.

The rest of the paper is organized as follows. Section 2 sets up the notation and presents the specific distributions and concepts from exterior differential systems (associated and retracting spaces) that are required in subsequent sections. The well-known results of [1] and [2] for driftless systems that were mentioned above are given in Section 3. Section 4 exhibits the proof of the main result of this work and its corollary, along with two examples illustrating its application.

\section{Mathematical Preliminaries}

The sets of natural and real numbers are denoted as $\mathbb{N}$ (where $0 \in \mathbb{N}$ ) and $\mathbb{R}$, respectively. Throughout the text, unless otherwise stated, $M$ is 
a finite-dimensional smooth manifold and $U \subset M$ is an open set. Then, $\mathcal{C}^{\infty}(U), \mathfrak{X}(U)$ and $\Omega^{r}(U)$ denote the set of smooth functions on $U$, the set of smooth vector fields on $U$ and the set of $r$-forms on $U$, respectively, for all $r \in \mathbb{N}$. Given $p \in M, T_{p} M$ and $T_{p}^{*} M$ are the tangent and cotangent spaces to $M$ at $p$, respectively, and $\Omega_{p}^{r} M$ is the set of alternating tensors of covariant order $r$ on $T_{p} M$. The set $\Omega_{p} M=\bigoplus_{r=0}^{\infty} \Omega_{p}^{r} M$ (direct sum) is the exterior algebra over $T_{p} M$, where $\Omega_{p}^{0} M=\mathbb{R}$, and $\Omega(U)=\bigoplus_{r=0}^{\infty} \Omega^{r}(U)$ is the exterior algebra on $U$, where $\Omega^{0}(U)=\mathcal{C}^{\infty}(U)$. Let $f \in \mathcal{C}^{\infty}(U), X, Y \in \mathfrak{X}(U)$, $\omega \in \Omega(U)$. Then: $L_{X} f \in \mathcal{C}^{\infty}(U)$ denotes the Lie derivative of $f$ with respect to $X,[X, Y] \in \mathfrak{X}(U)$ is the Lie bracket of $X$ and $Y, L_{X} \omega \in \Omega(U)$ is the Lie derivative of $\omega$ with respect to $X$, and $d \omega \in \Omega(U)$ is the exterior derivative of $\omega$. Let $X^{1}, \ldots, X^{r} \in \mathfrak{X}(U)$ and $V \subset U$. Given $q \in V,\left.\left\{X^{1}, \ldots, X^{r}\right\}\right|_{q}$ denotes $\left\{X_{q}^{1}, \ldots, X_{q}^{r}\right\}$, and when $X_{p}^{1}, \ldots, X_{p}^{r}$ are independent vectors for all $p \in V$, one simply says that $X^{1}, \ldots, X^{r}$ are independent on $V$. Similar conventions shall also be adopted for 1-forms.

Following [9], one considers in this work a distribution (respectively, codistribution) on $U$ simply as a submodule of $\mathfrak{X}(U)$ (resp., $\Omega^{1}(U)$ ). Let $\Delta$ be a distribution on $U$ and $p \in U$. Define $\Delta_{p}=\left\{X_{p} \in T_{p} M \mid X_{p}=\right.$ $Y_{p}$, where $\left.Y \in \Delta\right\}$. The concept of a regular point and the annihilator $\Delta^{\perp} \subset \Omega^{1}(U)$ of $\Delta$ are defined in the usual manner. One writes $\operatorname{dim}(\Delta)=r$ when $\operatorname{dim}\left(\Delta_{p}\right)=r$, for all $p \in U$. Given an open set $V \subset U$, define the distribution $\Delta \mid V=\operatorname{span}_{\mathcal{C}^{\infty}(V)}\{X \in \mathfrak{X}(V)|X=Y| V$, where $Y \in \Delta\}$. Note that $\{X \in \mathfrak{X}(V)|X=Y| V$, with $Y \in \Delta\}$ is not necessarily a distribution on $V$. It is clear that analogous concepts to the ones presented above for distributions can be readily defined for codistributions. Let $\Delta_{1}, \Delta_{2}, \Delta_{3}$ be distributions on $U$. Consider the distributions $\Delta_{1}+\Delta_{2}=$ $\left\{X \in \mathfrak{X}(U) \mid X=X^{1}+X^{2}\right.$, where $\left.X^{1} \in \Delta_{1}, X^{2} \in \Delta_{2}\right\}$ and $\left[\Delta_{1}, \Delta_{2}\right]=$ $\operatorname{span}_{\mathcal{C}^{\infty}(U)}\left\{X \in \mathfrak{X}(U) \mid X=\left[X^{1}, X^{2}\right]\right.$, where $\left.X^{1} \in \Delta_{1}, X^{2} \in \Delta_{2}\right\}$. Note that $\Delta_{1}+\left[\Delta_{2}, \Delta_{3}\right]=\operatorname{span}_{\mathcal{C}^{\infty}(U)}\left\{X \in \mathfrak{X}(U) \mid X=X^{1}+\left[X^{2}, X^{3}\right]\right.$, where $X^{i} \in$ $\left.\Delta_{i}, 1 \leq i \leq 3\right\}$. If $V \subset U$ is open, then $\left(\Delta_{2}+\Delta_{3}\right)\left|V=\Delta_{2}\right| V+\Delta_{3} \mid V$ and $\left(\Delta_{1}+\left[\Delta_{2}, \Delta_{3}\right]\right)\left|V=\Delta_{1}\right| V+\left[\Delta_{2}\left|V, \Delta_{3}\right| V\right]$.

Let $\Delta$ be a distribution on $U$. Define, for each $k \in \mathbb{N}$, the distributions $F_{0}=G_{0}=\Delta, F_{k+1}=F_{k}+\left[F_{k}, F_{0}\right] \supset F_{k}, G_{k+1}=G_{k}+\left[G_{k}, G_{k}\right] \supset G_{k} \supset F_{k}$. The sequences of distributions $\left(F_{k}\right)$ and $\left(G_{k}\right)$ are called the Lie flag and the derived flag of $\Delta$, respectively [10].

The next result, which is asserted in [1] and [9], follows by induction and the usual properties of the Lie bracket. 
Proposition 1. Let $\left(F_{k}\right)$ and $\left(G_{k}\right)$ be the Lie and derived flags of $\Delta=$ $\operatorname{span}_{\mathcal{C}^{\infty}(U)}\left\{X^{1}, X^{2}\right\}$, respectively, where $X^{1}, X^{2} \in \mathfrak{X}(U)$. Take $P_{0}=Q_{0}=$ $\left\{X^{1}, X^{2}\right\}$ and consider, for each $k \in \mathbb{N}$,

$$
\begin{aligned}
& P_{k+1}=\left\{X \in \mathfrak{X}(U) \mid X=\left[Y^{k+2},\left[Y^{k+1},\left[\ldots,\left[Y^{2}, Y^{1}\right] \ldots\right]\right]\right.\right. \\
&\text { where } \left.Y^{1}, \ldots, Y^{k+2} \in P_{0}\right\}, \\
& Q_{k+1}=\left\{X \in \mathfrak{X}(U) \mid X=\left[Y^{1}, Y^{2}\right], \text { where } Y^{1}, Y^{2} \in \cup_{j=0}^{k} Q_{j}\right\} \subset Q_{k+2} .
\end{aligned}
$$

Then, $F_{0}=G_{0}=\Delta$ and, for each $k \in \mathbb{N}$,

$$
\begin{aligned}
F_{k+1} & =\operatorname{span}_{\mathcal{C}^{\infty}(U)}\left(\cup_{j=0}^{k+1} P_{j}\right)=F_{k}+\operatorname{span}_{\mathcal{C}^{\infty}(U)}\left(P_{k+1}\right), \\
G_{k+1} & =G_{0}+\operatorname{span}_{\mathcal{C}^{\infty}(U)}\left(Q_{k+1}\right)=G_{k}+\operatorname{span}_{\mathcal{C}^{\infty}(U)}\left(Q_{k+1}\right) .
\end{aligned}
$$

Remark 1. It is clear that $F_{0}=G_{0}=\operatorname{span}\left\{X^{1}, X^{2}\right\}, F_{1}=G_{1}=\operatorname{span}\left\{X^{1}, X^{2}\right.$, $\left.\left[X^{1}, X^{2}\right]\right\}, F_{2}=G_{2}=\operatorname{span}\left\{X^{1}, X^{2},\left[X^{1}, X^{2}\right],\left[X^{1},\left[X^{1}, X^{2}\right]\right],\left[X^{2},\left[X^{1}, X^{2}\right]\right]\right\}$. However, $F_{k}$ and $G_{k}$ are not necessarily identical when $k \geq 3$.

Remark 2. Consider system (1) and take $X^{1}=g_{1}, X^{2}=g_{2}$. Let $\left(F_{k}\right)$ and $\left(G_{k}\right)$ be the Lie and derived flags of $\Delta=\operatorname{span}_{\mathcal{C}^{\infty}(U)}\left\{g_{1}, g_{2}\right\}$, respectively. It is immediately verified by induction that $\left(F_{k}\right)$ and $\left(G_{k}\right)$ are invariant under regular static state feedbacks. More precisely, suppose that $u=\alpha+\beta v$ is such a feedback defined on $U$. Let $\widehat{\Delta}=\operatorname{span}_{\mathcal{C}^{\infty}(U)}\left\{\widehat{g}_{1}, \widehat{g}_{2}\right\}$, where $\widehat{g}_{i}=\beta_{i 1} g_{1}+\beta_{i 2} g_{2}$, $i=1,2$, and let $\left(\widehat{F}_{k}\right)$ and $\left(\widehat{G}_{k}\right)$ be the Lie and derived flags of $\widehat{\Delta}$, respectively. Then, $F_{k}=\widehat{F}_{k}, G_{k}=\widehat{G}_{k}$, for $k \in \mathbb{N}$.

Let $p \in M$ and $X_{p} \in T_{p} M$. The interior product with $X_{p}$ is the map $i_{X_{p}}: \Omega_{p}^{r} M \rightarrow \Omega_{p}^{r-1} M$ defined as $\left(i_{X_{p}} \omega_{p}\right)\left(Y_{p}^{1}, \ldots, Y_{p}^{r-1}\right)=\omega_{p}\left(X_{p}, Y_{p}^{1}, \ldots, Y_{p}^{r-1}\right)$, for all $\omega_{p} \in \Omega_{p}^{r} M$ and all $Y_{p}^{1}, \ldots, Y_{p}^{r-1} \in T_{p} M$, where $r \in \mathbb{N}, \Omega_{p}^{-1} M \triangleq\{0\}$ and $i_{X_{p}} \omega_{p} \triangleq 0$, for all $\omega_{p} \in \Omega_{p}^{0} M=\mathbb{R}$. Extending it by linearity, one obtains the mapping $i_{X_{p}}: \Omega_{p} M \rightarrow \Omega_{p} M$. One also denotes $i_{X_{p}} \omega_{p}$ as $\left.X_{p}\right\lrcorner \omega_{p}$. The map $i_{X_{p}}$ is linear, satisfies $i_{X_{p}} \circ i_{X_{p}}=0$ and is an antiderivation, that is, if $\theta_{p} \in \Omega_{p}^{r} M$ and $\sigma_{p} \in \Omega_{p}^{s} M$, then $\left.\left.\left.X_{p}\right\lrcorner\left(\theta_{p} \wedge \sigma_{p}\right)=\left(X_{p}\right\lrcorner \theta_{p}\right) \wedge \sigma_{p}+(-1)^{r} \theta_{p} \wedge\left(X_{p}\right\lrcorner \sigma_{p}\right)[9]$. For the purposes of this work, it suffices to consider the definition of associated and retracting spaces given in the sequel. For a more general notion (but equivalent in the present context) concerning (homogeneous) algebraic ideals, see [11], [9], [12]. Let $\Lambda=\operatorname{span}_{\mathcal{C}^{\infty}(U)}\left\{\lambda^{1}, \ldots, \lambda^{m}\right\}$ be a codistribution on $U$. One defines the associated space or Cauchy characteristic space 
determined by $\Lambda$ at $p \in U$ as

$$
\begin{array}{r}
A(\Lambda)_{p}=\left\{X_{p} \in T_{p} M \mid X_{p}\right\lrcorner d \lambda_{p}^{i} \in \Lambda_{p}=\operatorname{span}_{\mathbb{R}}\left\{\lambda_{p}^{1}, \ldots, \lambda_{p}^{m}\right\} \\
\text { and } \left.\left\langle\lambda_{p}^{i}, X_{p}\right\rangle=0, \text { for } 1 \leq i \leq m\right\},
\end{array}
$$

and the retracting space determined by $\Lambda$ at $p \in U$ as $C(\Lambda)_{p}=\left(A(\Lambda)_{p}\right)^{\perp}$. Note that $A(\Lambda)_{p}$ and $C(\Lambda)_{p}$ are subspaces of $T_{p} M$ and $T_{p}^{*} M$, respectively. Furthermore, given an open set $V \subset U$ and $p \in V$, one has $A(\Lambda)_{p}=A(\Lambda \mid V)_{p}$.

\section{Known Results for Driftless Systems}

Theorem 1 and Theorem 2 given below are the well-known results for driftless systems with two inputs mentioned in the introduction. They will be used in the results established in the next section. Note that Theorem 2 is a generic result.

Theorem 1. [1] Consider system (11) with $f=0$. Define $\Delta=\operatorname{span}_{\mathcal{C}_{(U)}}\left\{g_{1}, g_{2}\right\}$ and $\Lambda=\Delta^{\perp}$. Let $\left(F_{k}\right)$ and $\left(G_{k}\right)$ be the Lie and derived flags of $\Delta$, respectively. Given $p \in U$, the following assertions are equivalent:

1) For each $0 \leq k \leq n-2, p \in U$ is a regular point of $F_{k}, G_{k}$ with $\operatorname{dim}\left(F_{k}(p)\right)=\operatorname{dim}\left(G_{k}(p)\right)=2+k$

2) There exists a change of coordinates $z=\varphi(x)$ and a regular static state feedback $u=\beta v$, both of them defined on an open neighborhood $V \subset U$ of $p$, in which the expression of the resulting closed-loop system in the coordinates $z=\left(z_{1}, \ldots, z_{n}\right)$ is given by (2) .

Theorem 2. 2] Consider system (11) with $f=0$. Let $\left(G_{k}\right)$ be the derived flag of $\Delta=\operatorname{span}_{\mathcal{C}^{\infty}(U)}\left\{g_{1}, g_{2}\right\}$ and suppose that $\operatorname{dim}\left(G_{k}(q)\right)=2+k$, for all $0 \leq k \leq n-2, q \in D$, where $D \subset U$ is an open dense set in $U$. Then, there exists an open and dense set $V$ in $U$ such that, for every $p \in V$, property 2) above holds. In particular, for every $p \in V \subset U$, property 1) is true.

\section{Main Result}

The proof of Theorem 3, which is the main result of this paper, relies on the lemmas given below. 
Lemma 1. Let $M$ be a manifold with $\operatorname{dim}(M)=n \geq 4$. Assume that $z=\left(z_{1}, \ldots, z_{n}\right)$ is a local chart of $M$ defined on an open set $U$. Consider the following codistributions on $U$

$$
\Lambda^{k}=\operatorname{span}_{\mathcal{C}^{\infty}(U)}\left\{d z_{1}-z_{2} d z_{n}, \ldots, d z_{n-2-k}-z_{n-1-k} d z_{n}\right\}
$$

for each $1 \leq k \leq n-3$. Then, $C_{q}^{k}=\left.\operatorname{span}_{\mathbb{R}}\left\{d z_{1}, \ldots, d z_{n-1-k}, d z_{n}\right\}\right|_{q} \supset \Lambda_{q}^{k}$, for all $q \in U, 1 \leq k \leq n-3$, where $C_{q}^{k} \triangleq C\left(\Lambda^{k}\right)_{q}$ is the retracting space determined by $\Lambda^{k}$ at $q \in U$.

Proof. Let $q \in U, 1 \leq k \leq n-3$ and $A_{q}^{k}=A\left(\Lambda^{k}\right)_{q}$. Then $S_{q}=\left\{\partial / \partial z_{n-1-k}, \ldots\right.$, $\left.\partial / \partial z_{n-1}, \gamma\right\}\left.\right|_{q}$ is a basis of $\left(\Lambda_{q}^{k}\right)^{\perp}$, where $\gamma=z_{2} \partial / \partial z_{1}+\cdots+z_{n-1-k} \partial / \partial z_{n-2-k}+$ $\partial / \partial z_{n}$. Let $X_{q} \in T_{q}(M)$. Then, $X_{q} \in A_{q}^{k}$ if and only if $X_{q} \in \operatorname{span}_{\mathbb{R}}\left(S_{q}\right)$ and $\left.X_{q}\right\lrcorner\left.\left(d z_{j} \wedge d z_{n}\right)\right|_{q} \in \Lambda_{q}^{k}$ for $2 \leq j \leq n-1-k$, which in turn is equivalent to $\left.X_{q} \in \operatorname{span}_{\mathbb{R}}\left\{\partial / \partial z_{n-k}, \ldots, \partial / \partial z_{n-1}\right\}\right|_{q}$. This completes the proof.

Lemma 2. Let $U \subset M$ be open with $\operatorname{dim}(M)=n \geq 3$. Consider that $z=$ $\left(z_{1}, \ldots, z_{n}\right)$ is a local chart defined on $U$ such that $X^{1}, X^{2} \in \mathfrak{X}(U)$ are respectively described as $X^{1}(z)=\left(z_{2}, \ldots, z_{n-1}, 0,1\right), X^{2}(z)=(0, \ldots, 0,1,0)$ (note that $\left.X^{2}=\partial / \partial z_{n-1}\right)$. Let $\left(F_{k}\right)$ and $\left(G_{k}\right)$ be the Lie and derived flags of $\Delta=$ $\operatorname{span}_{\mathcal{C}^{\infty}(U)}\left\{X^{1}, X^{2}\right\}$, respectively. Then, $F_{k}=G_{k}=\operatorname{span}_{\mathcal{C}^{\infty}(U)}\left\{X^{1}, \partial / \partial z_{n-1}\right.$, $\left.\ldots, \partial / \partial z_{n-1-k}\right\}$ and $\operatorname{dim}\left(F_{k}\right)=\operatorname{dim}\left(G_{k}\right)=2+k$, for $0 \leq k \leq n-2$.

Proof. One begins by showing by induction on $0 \leq k \leq n-2$ that

$$
G_{k}=\operatorname{span}_{\mathcal{C}^{\infty}(U)}\left\{X^{1}, \partial / \partial z_{n-1}, \ldots, \partial / \partial z_{n-1-k}\right\} .
$$

Note that $G_{0}=\operatorname{span}_{\mathcal{C}^{\infty}(U)}\left\{X^{1}, \partial / \partial z_{n-1}\right\}$. Consider the induction hypothesis: $G_{k}=\operatorname{span}_{\mathcal{C}^{\infty}(U)}\left\{X^{1}, \partial / \partial z_{n-1}, \ldots, \partial / \partial z_{n-1-k}\right\}$, where $0 \leq k \leq n-3$. Define $H=G_{k}+\operatorname{span}_{\mathcal{C}^{\infty}(U)}\left\{\partial / \partial z_{n-1-(k+1)}\right\}$. It suffices to prove that $G_{k+1}=H$. By hypothesis, $X^{1}(z)=\left(z_{2}, \ldots, z_{n-1}, 0,1\right)$. It is easy to see that 2

$$
\left[\partial / \partial z_{n-j}, X^{1}\right]=\partial / \partial z_{n-1-j} \in F_{j} \subset G_{j}, \quad \text { for } 1 \leq j \leq n-2 .
$$

In particular, $\partial / \partial z_{n-1-(k+1)}=\partial / \partial z_{n-2-k} \in G_{k+1}$. Hence, $H \subset G_{k+1}$ because $G_{k} \subset G_{k+1}$. Now, let $Y=Y^{1}+\left[Y^{2}, Y^{3}\right] \in G_{k+1}$, where $Y^{i} \in G_{k}$, for $1 \leq i \leq 3$. One has $Y^{i}=\alpha_{i} X^{1}+\sum_{j=1}^{k+1} \beta_{i j} \partial / \partial z_{n-j}$, where $\alpha_{i}, \beta_{i j} \in \mathcal{C}^{\infty}(U)$,

\footnotetext{
${ }^{2}$ See also [9, Lemma 11.8].
} 
for $1 \leq i \leq 3$. It follows from (5) that $\left[Y^{2}, Y^{3}\right]=\left[\alpha_{1} X^{1}+\sum_{j=1}^{k+1} \beta_{1 j} \partial / \partial z_{n-j}\right.$, $\left.\alpha_{2} X^{1}+\sum_{j=1}^{k+1} \beta_{2 j} \partial / \partial z_{n-j}\right]=\lambda X^{1}+\sum_{j=1}^{k+1} \gamma_{j} \partial / \partial z_{n-j}+\sum_{j=1}^{k+1} \delta_{j} \partial / \partial z_{n-1-j} \in H$, where $\lambda, \gamma_{j}, \delta_{j} \in \mathcal{C}^{\infty}(U)$. Since $Y^{1} \in G_{k} \subset H$, one has $Y \in H$. Thus, $G_{k+1} \subset$ $H$, and hence $G_{k+1}=H$. Finally, recall that $F_{k} \subset G_{k}$, for $0 \leq k \leq n-2$, with $F_{0}=G_{0}=\Delta$. On the other hand, it is immediate from (44) and (5) that $G_{k} \subset F_{k}$, for $1 \leq k \leq n-2$. Therefore, $F_{k}=G_{k}, 0 \leq k \leq n-2$.

Theorem 3. Consider system (1) and let $\left(F_{k}\right)$ and $\left(G_{k}\right)$ be the Lie and derived flags of $\Delta=\operatorname{span}_{\mathcal{C}^{\infty}(U)}\left\{g_{1}, g_{2}\right\}$, respectively. For each $1 \leq k \leq n-3$, define $\Lambda^{k}=\left(G_{k}\right)^{\perp}$ and $C_{q}^{k}=C\left(\Lambda^{k}\right)_{q}$, for $q \in U$. Let $p \in U$. Then, there exist a change of coordinates $z=\varphi(x)$ and a regular static state feedback of the form $u=\alpha+\beta v$, both of them defined on an open neighborhood $V \subset U$ of $p$, such that the expression of the resulting closed-loop system in the coordinates $z=\left(z_{1}, \ldots, z_{n}\right)$ is given by the triangular form (3) if and only if there exists an open neighborhood $W \subset U$ of $p$ in which the following geometric conditions are satisfied:

1) $\operatorname{dim}\left(F_{k}(q)\right)=\operatorname{dim}\left(G_{k}(q)\right)=2+k$, for $0 \leq k \leq n-2, q \in W$;

2) $L_{f} \omega_{q} \in C_{q}^{k}$, for $1 \leq k \leq n-3, q \in W, \omega \in \Lambda^{k}$.

Remark 3. Note that the control vector fields in (21) and (3i) are exactly the same, that condition 1) above is precisely assertion 1) in Theorem 1 for driftless systems, and that the drift $f$ in (1) is taken into account by means of condition 2). Assume that both conditions of Theorem 3 are met. It can be seen from the sufficiency part of the proof of Theorem 3 that the required change of coordinates $z=\varphi(x)$ and $\beta(x)$ in the regular feedback $u=\alpha+\beta v$ may always be taken as the ones provided by Theorem 1 (except for a possible restriction of their domain of definition). This means that, in order for (1) to be described by (3), it suffices to obtain a change of coordinates $z=\varphi(x)$ and a feedback $\beta(x)$ such that the controls vector fields $g_{1}, g_{2}$ in (1) are described by the chained form (21) with $z=\varphi(x)$ and $u=\beta v$. The existence of such $z=\varphi(x)$ and $u=\beta v$ is ensured by condition 1) of Theorem 3, and in general they may be constructed by the techniques given in [13, Algorithm 1] and [10, Section 3]. Another approach is the result in [14, Proposition 7]. Having obtained the referred $z=\varphi(x)$ and $u=\beta v$, the proof of Theorem 3 shows that condition 2) ensures that after applying the regular feedback $u=\alpha+\beta v$ in (11), where $\alpha=\beta \bar{\alpha}$ and $\bar{\alpha}=-\left(\left\langle d z_{n}, f\right\rangle,\left\langle d z_{n-1}, f\right\rangle\right)$ (cf. (15) and (18)), one has that the closed-loop drift $f+\alpha_{1} g_{1}+\alpha_{2} g_{2}$ exhibits the triangular structure in (3) in the coordinates $z=\varphi(x)$. Thus, (11) has been transformed into (3). 
Before proving Theorem 3, one explains the conventions adopted in its proof. Let $\Delta$ a distribution on $U \subset M$ and $X^{1}, \ldots, X^{r} \in \mathfrak{X}(U)$. If $\Delta=$ $\operatorname{span}_{\mathcal{C}^{\infty}(U)}\left\{X^{1}, \ldots, X^{r}\right\}$, then one simply writes $\Delta=\operatorname{span}\left\{X^{1}, \ldots, X^{r}\right\}$. Let $V, W \subset U$ be open sets with $V \subset W \subset U$ and $Y^{1}, \ldots, Y^{m} \in \mathfrak{X}(W)$. In case $\Delta \mid V=\operatorname{span}_{\mathcal{C}^{\infty}(V)}\left\{Y^{1}\left|V, \ldots, Y^{m}\right| V\right\}$, then one writes $\Delta \mid V=\operatorname{span}\left\{Y^{1}, \ldots\right.$, $\left.Y^{m}\right\}$ over $\mathcal{C}^{\infty}(V)$. Analogous notations for codistributions will also be used.

Proof. The result is shown for $n \geq 4$. The reader will have no difficulty in verifying that the arguments presented also assure its validity for $2 \leq n \leq 3$. One begins by proving necessity.

(Necessity) Consider the resulting closed-loop vector fields on $W=V$ :

$$
\begin{aligned}
& \widehat{f}=f+\alpha_{1} g_{1}+\alpha_{2} g_{2} \in \mathfrak{X}(W), \\
& \widehat{g}_{1}=\beta_{11} g_{1}+\beta_{12} g_{2}, \quad \widehat{g}_{2}=\beta_{21} g_{1}+\beta_{22} g_{2} \in G_{0} \mid W,
\end{aligned}
$$

with $\beta=\left(\beta_{i j}\right)$. The expressions of $\widehat{f}, \widehat{g}_{1}, \widehat{g}_{2}$ in the coordinates $z=\left(z_{1}, \ldots, z_{n}\right)$ are respectively given as

$$
\begin{aligned}
& \widehat{f}(z)=\left(\phi_{1}(z), \ldots, \phi_{n-2}(z), 0,0\right), \\
& \widehat{g}_{1}(z)=\left(z_{2}, \ldots, z_{n-1}, 0,1\right), \\
& \widehat{g}_{2}(z)=(0, \ldots, 0,1,0),
\end{aligned}
$$

where $\phi_{1}, \ldots, \phi_{n-2}$ are as in (3) $)$. Let $\left(\widehat{F}_{k}\right)$ and $\left(\widehat{G}_{k}\right)$ be the Lie and the derived flags of $\widehat{\Delta}=\operatorname{span}_{\mathcal{C}^{\infty}(W)}\left\{\widehat{g}_{1}, \widehat{g}_{2}\right\}$, respectively. According to Lemma 2 , $\widehat{F}_{k}=\widehat{G}_{k}=\operatorname{span}_{\mathcal{C}^{\infty}(W)}\left\{\widehat{g}_{1}, \partial / \partial z_{n-1}, \partial / \partial z_{n-2}, \ldots, \partial / \partial z_{n-1-k}\right\}$ with $\operatorname{dim}\left(\widehat{F}_{k}\right)=$ $\operatorname{dim}\left(\widehat{G}_{k}\right)=2+k$, for $0 \leq k \leq n-2$, and $\widehat{g}_{2}=\partial / \partial z_{n-1}$. Since $\beta=\left(\beta_{i j}\right)$ is invertible on $W$, one concludes from Remark 2 that $F_{k} \mid W=\widehat{F}_{k}=\widehat{G}_{k}=$ $G_{k} \mid W$ with $\operatorname{dim}\left(F_{k} \mid W\right)=\operatorname{dim}\left(G_{k} \mid W\right)=2+k$, for every $0 \leq k \leq n-2$. Define $\theta_{i}=d z_{i}-z_{i+1} d z_{n}$, for $1 \leq i \leq n-3$. Fix $1 \leq i \leq n-3$. By restricting $W$ if necessary, one has that

$$
\Lambda^{n-2-i} \mid W=\left(G_{n-2-i} \mid W\right)^{\perp}=\operatorname{span}\left\{\theta_{1}, \ldots, \theta_{i}\right\} \subset\left(G_{0} \mid W\right)^{\perp}
$$

over $\mathcal{C}^{\infty}(W)$. Therefore, using Lemma 1,

$$
C_{q}^{n-2-i}=\left.\operatorname{span}_{\mathbb{R}}\left\{d z_{1}, \ldots, d z_{i+1}, d z_{n}\right\}\right|_{q} \supset \Lambda_{q}^{n-2-i}, \quad \text { for } q \in W .
$$

From (7), one gets $L_{\widehat{f}} \theta_{i}=d \phi_{i}-\phi_{i+1} d z_{n}, L_{\widehat{g}_{1}} \theta_{i}=d z_{i+1}-z_{i+2} d z_{n}, L_{\widehat{g}_{2}} \theta_{i}=$ 0 , with $d \phi_{i} \in \operatorname{span}_{\mathcal{C}^{\infty}(W)}\left\{d z_{1}, d z_{2}, \ldots, d z_{i+1}, d z_{n}\right\}$. By (9) $,\left(L_{\widehat{f}} \theta_{i}\right)_{q},\left(L_{\widehat{g}_{1}} \theta_{i}\right)_{q}$, 
$\left(L_{\widehat{g}_{2}} \theta_{i}\right)_{q} \in C_{q}^{n-2-i}$, for $q \in W$. Let $q \in W, \omega \in \Lambda^{n-2-i}, \bar{\omega}=\omega \mid W$. Then (8) and (9) imply $L_{\widehat{f}} \bar{\omega}_{q}, L_{\widehat{g}_{1}} \bar{\omega}_{q}, L_{\widehat{g}_{2}} \bar{\omega}_{q} \in C_{q}^{n-2-i}$, and (다) gives that $L_{f} \bar{\omega}_{q} \in C_{q}^{n-2-i}$ since $\left\langle\bar{\omega}, \widehat{g}_{1}\right\rangle=\left\langle\bar{\omega}, \widehat{g}_{2}\right\rangle=0$ by (8) again.

(Sufficiency) By hypothesis, $p$ is a regular point of $F_{k}, G_{k}$ with $\operatorname{dim}\left(F_{k}(p)\right)=$ $\operatorname{dim}\left(G_{k}(p)\right)=2+k$, for $0 \leq k \leq n-2$. Hence Theorem 1 establishes that, by restricting $W$ if necessary, there exists a change of coordinates $z=\varphi(x)$ and regular static state feedback $u=\beta v$, both defined on $W$, such that the expressions of $f$,

$$
\widehat{g}_{1}=\beta_{11} g_{1}+\beta_{12} g_{2}, \quad \widehat{g}_{2}=\beta_{21} g_{1}+\beta_{22} g_{2},
$$

in the coordinates $z=\left(z_{1}, \ldots, z_{n}\right)$, are respectively given as

$$
\begin{aligned}
& f(z)=\left(\gamma_{1}(z), \ldots, \gamma_{n}(z)\right), \\
& \widehat{g}_{1}(z)=\left(z_{2}, \ldots, z_{n-1}, 0,1\right), \\
& \widehat{g}_{2}(z)=(0, \ldots, 0,1,0) .
\end{aligned}
$$

It can be assumed that $\frac{3}{3} \beta=\widetilde{\beta} \mid W$, where $\widetilde{\beta}=\left(\widetilde{\beta}_{i j}\right): U \rightarrow \mathbb{R}^{2 \times 2}$ is smooth.

Since $\beta=\left(\beta_{i j}\right)$ is invertible on $W$, the proof of necessity above provides, by restricting $W$ if necessary, that

$$
G_{k} \mid W=\operatorname{span}\left\{\widehat{g}_{1}, \partial / \partial z_{n-1}, \ldots, \partial / \partial z_{n-1-k}\right\}
$$

with $\widehat{g}_{2}=\partial / \partial z_{n-1}, \operatorname{dim}\left(G_{k} \mid W\right)=2+k$, for $1 \leq k \leq n-2$, and

$$
\Lambda^{n-2-i} \mid W=\left(G_{n-2-i} \mid W\right)^{\perp}=\operatorname{span}\left\{\theta_{1}, \ldots, \theta_{i}\right\}
$$

over $\mathcal{C}^{\infty}(W)$, for $1 \leq i \leq n-3$, where $\theta_{i}=d z_{i}-z_{i+1} d z_{n}$. Hence, (13) and Lemma 1 give

$$
C_{q}^{n-2-i}=\left.\operatorname{span}_{\mathbb{R}}\left\{d z_{1}, \ldots, d z_{i+1}, d z_{n}\right\}\right|_{q} \supset \Lambda_{q}^{n-2-i}, q \in W, 1 \leq i \leq n-3 .
$$

Consider the following maps on $W$ (see (11)):

$$
\begin{aligned}
& \bar{\alpha}=\left(\bar{\alpha}_{1}, \bar{\alpha}_{2}\right) \triangleq-\left(\left\langle d z_{n}, f\right\rangle,\left\langle d z_{n-1}, f\right\rangle\right)=-\left(\gamma_{n}, \gamma_{n-1}\right), \\
& \widehat{f}=f+\bar{\alpha}_{1} \widehat{g}_{1}+\bar{\alpha}_{2} \widehat{g}_{2}, \\
& \phi_{i}=\left\langle d z_{i}, \widehat{f}\right\rangle, \quad \text { for } 1 \leq i \leq n-2 .
\end{aligned}
$$

\footnotetext{
${ }^{3}$ This follows by using an adequate bump function and by restricting $W$ if necessary.
} 
By construction,

$$
\left\langle d z_{n}, \widehat{f}\right\rangle=\left\langle d z_{n-1}, \widehat{f}\right\rangle=0 .
$$

Indeed, $\left\langle d z_{n}, \widehat{g}_{1}\right\rangle=\left\langle d z_{n-1}, \widehat{g}_{2}\right\rangle=1,\left\langle d z_{n-1}, \widehat{g}_{1}\right\rangle=\left\langle d z_{n}, \widehat{g}_{2}\right\rangle=0$ (cf. (11)) . Thus, $\left\langle d z_{n-j}, \widehat{f}\right\rangle=\gamma_{n-j}-\gamma_{n-j}=0$, for $0 \leq j \leq 1$.

Now, because $\widehat{f}(z)=\left(\phi_{1}(z), \ldots, \phi_{n-2}(z), 0,0\right)$, it remains to show that

$$
\left.\left.d \phi_{i}\right|_{q} \in \operatorname{span}_{\mathbb{R}}\left\{d z_{1}, \ldots, d z_{i+1}, d z_{n}\right\}\right|_{q}, \quad \text { for } q \in W, 1 \leq i \leq n-3 .
$$

Fix $1 \leq i \leq n-3, q \in W$. By (11), $L_{\widehat{g}_{1}} \theta_{i}=d z_{i+1}-z_{i+2} d z_{n}$ and $L_{\widehat{g}_{2}} \theta_{i}=0$, and $L_{\widehat{f}} \theta_{i}=d \phi_{i}-\phi_{i+1} d z_{n}$ from (16). It can be assumed, by restricting $W$ if necessary, that $\bar{\alpha}=\widetilde{\alpha} \mid W$ and $\theta_{i}=\widetilde{\theta}_{i} \mid W$, where $\widetilde{\alpha}=\left(\widetilde{\alpha}_{1}, \widetilde{\alpha}_{2}\right), \widetilde{\alpha}_{1}, \widetilde{\alpha}_{2} \in \mathcal{C} \infty(U)$, and $\tilde{\theta}_{i} \in \Lambda^{n-2-i}=\left(G_{n-2-i}\right)^{\perp}$. Since $\beta=\widetilde{\beta} \mid W, G_{0} \subset G_{k}(k \in \mathbb{N})$ and $\widetilde{\theta}_{i} \in \Lambda^{n-2-i}$, it follows from condition 2) in Theorem 3, (10), (14) and (15) that $\left(L_{\widehat{f}} \theta_{i}\right)_{q}=\left(L_{\widehat{f}} \widetilde{\theta}_{i}\right)_{q} \in C_{q}^{n-2-i}$. Hence, (17) holds.

Finally, let $v=\left(v_{1}, v_{2}\right) \in \mathbb{R}^{2}$ and define $X^{v}=\widehat{f}+\widehat{g}_{1} v_{1}+\widehat{g}_{2} v_{2} \in \mathfrak{X}(W)$. By (11), (15), (16) and (17), the expression of $X^{v}$ in the coordinates $z=$ $\left(z_{1}, \ldots, z_{n}\right)$ on $W$ is given by (3) , by restricting $W$ to an open connected set if necessary. Therefore,

$$
u=\alpha+\beta v=\beta \bar{\alpha}+\beta v, \quad \text { with } \bar{\alpha}=-\left(\left\langle d z_{n}, f\right\rangle,\left\langle d z_{n-1}, f\right\rangle\right),
$$

is the desired regular feedback, because $\widehat{f}=f+\bar{\alpha}_{1} \widehat{g}_{1}+\bar{\alpha}_{2} \widehat{g}_{2}, \widehat{g}_{1}=\beta_{11} g_{1}+\beta_{12} g_{2}$, $\widehat{g}_{2}=\beta_{21} g_{1}+\beta_{22} g_{2}, \bar{\alpha}=\left(\bar{\alpha}_{1}, \bar{\alpha}_{2}\right)$ and $\beta=\left(\beta_{i j}\right)$.

By using Theorem 2, one obtains the generic result:

Corollary 1. Consider system (1) and define $G_{k}, \Lambda^{k}, C_{q}^{k}$ as in Theorem 3 . Suppose that there exists an open dense set $D$ in $U$ such that:

1) $\operatorname{dim}\left(G_{k}(q)\right)=2+k$, for $0 \leq k \leq n-2, q \in D$;

2) $L_{f} \omega_{q} \in C_{q}^{k}$, for $1 \leq k \leq n-3, q \in D, \omega \in \Lambda^{k}$.

Then, there exists an open dense set $V$ in $U$ such that, for all $p \in V$, there exists a change of coordinates $z=\varphi(x)$ and a regular static state feedback of the form $u=\alpha+\beta v$, both defined on an open neighborhood $W \subset U$ of $p$, in which the expression of the resulting closed-loop system in the coordinates $z=\left(z_{1}, \ldots, z_{n}\right)$ is given by $(\underline{3})$.

One exhibits in the sequel two examples of application of Theorem 3 , 
Example 1. Consider system (1) with $U=\mathbb{R}^{4}, x=\left(x_{1}, \ldots, x_{4}\right) \in \mathbb{R}^{4}$ and

$$
\begin{aligned}
& f(x)=\left(0, x_{1}^{2}+x_{2}, 1, x_{1} x_{4}\right), \\
& g_{1}(x)=\left(x_{4}^{2}+1,\left(x_{3}-2 x_{1}\right)\left(x_{4}^{2}+1\right), 0,\left(x_{1}^{2}+x_{2}\right)\left(x_{4}^{2}+1\right)\right), \\
& g_{2}(x)=(0,0,1,0) .
\end{aligned}
$$

By relying on Remark 3, it will be shown that such system can be described by (3i) around every point of $\mathbb{R}^{4}$. One has

$$
\begin{aligned}
& g_{3}(x)=\left[g_{1}, g_{2}\right](x)=\left(0,-\left(x_{4}^{2}+1\right), 0,0\right), \\
& g_{4}(x)=\left[g_{1}, g_{3}\right](x)=\left(0,-2 x_{4}\left(x_{1}^{2}+x_{2}\right)\left(x_{4}^{2}+1\right), 0,\left(x_{4}^{2}+1\right)^{2}\right), \\
& g_{5}(x)=\left[g_{2}, g_{3}\right](x)=0,
\end{aligned}
$$

for $x \in \mathbb{R}^{4}$. According to Remark 1, $\operatorname{dim}\left(F_{k}\right)=\operatorname{dim}\left(G_{k}\right)=2+k$, for $0 \leq k \leq 2$. Since $G_{1}=\operatorname{span}_{\mathcal{C}^{\infty}\left(\mathbb{R}^{4}\right)}\left\{g_{1}, g_{2}, g_{3}\right\}$, it is straightforward to obtain

$$
\begin{aligned}
& \Lambda^{1}=\left(G_{1}\right)^{\perp}=\operatorname{span}_{\mathcal{C}^{\infty}\left(\mathbb{R}^{4}\right)}\{\gamma\}, \quad \text { where } \gamma=\left(x_{1}^{2}+x_{2}\right) d x_{1}-d x_{4}, \\
& C_{p}^{1}=\left.\operatorname{span}_{\mathbb{R}}\left\{d x_{1}, d x_{2}, d x_{4}\right\}\right|_{p} \supset \Lambda_{p}^{1},
\end{aligned}
$$

for $p \in \mathbb{R}^{4}$. It is easy to verify that $L_{f} \gamma_{p}=\left.\left[\left(x_{1}^{2}+x_{2}-x_{4}\right) d x_{1}-x_{1} d x_{4}\right]\right|_{p} \in C_{p}^{1}$, for $p \in \mathbb{R}^{4}$. Using $\Lambda_{p}^{1} \subset C_{p}^{1}$, one concludes that $L_{f} \omega_{p} \in C_{p}^{1}$, for $\omega \in \Lambda^{1}$, $p \in \mathbb{R}^{4}$, that is, one has shown that the conditions in Theorem 3 are met on $\mathbb{R}^{4}$. The (global) change of coordinates $z=\varphi(x)=\left(x_{4}, x_{1}^{2}+x_{2}, x_{3}, x_{1}\right)$ transforms $f(x), g_{1}(x), g_{2}(x)$ into $f(z)=\left(z_{1} z_{4}, z_{2}, 1,0\right), g_{1}(z)=\left(z_{2}\left(z_{1}^{2}+\right.\right.$ $\left.1), z_{3}\left(z_{1}^{2}+1\right), 0, z_{1}^{2}+1\right), g_{2}(z)=(0,0,1,0)$. The obvious choice for $\beta$ in the feedback $u=\beta v$ is then $\beta=\left(\beta_{i j}\right)=\operatorname{diag}\left(\left(z_{1}^{2}+1\right)^{-1}, 1\right)=\operatorname{diag}\left(\left(x_{4}^{2}+1\right)^{-1}, 1\right) \in$ $\mathbb{R}^{2 \times 2}$, since it yields $\widehat{g}_{1}(z)=\left(z_{1}^{2}+1\right)^{-1} g_{1}(z)=\left(z_{2}, z_{3}, 0,1\right), \widehat{g}_{2}(z)=g_{2}(z)=$ $(0,0,1,0)$. It remains to specify $\alpha$ in $u=\alpha+\beta v$. Following Remark 3, one takes $\alpha=\beta \bar{\alpha}$, where $\bar{\alpha}=-\left(\left\langle d z_{4}, f\right\rangle,\left\langle d z_{3}, f\right\rangle\right)=(0,-1)$.

Hence, after applying the (global) feedback $u=\alpha+\beta v$, the closed-loop system in the coordinates $z=\left(z_{1}, z_{2}, z_{3}, z_{4}\right)$ has the form (3) with

$$
\dot{z}=\left(z_{1} z_{4}, z_{2}, 0,0\right)+\left(z_{2}, z_{3}, 0,1\right) v_{1}+(0,0,1,0) v_{2} .
$$

Note that $y=\left(z_{1}, z_{4}\right)=\left(x_{4}, x_{1}\right)$ is a flat output around the points in which $v_{1} \neq 0$.

\footnotetext{
${ }^{4} \mathrm{~A}$ computational procedure for determining $C_{p}^{1}$ is outlined in the proof of Lemma 1
} 
Although the present academic example has codimension $n-2=2$, it does not satisfy the sufficient geometric conditions for flatness established in [5, p. 266]. Indeed, $\left[g_{1}, g_{2}\right] \notin \operatorname{span}\left\{g_{1}, g_{2}\right\},\left[g_{1}, g_{2}\right] \notin \operatorname{span}\left\{g_{1}, \operatorname{ad}_{f} g_{1}\right\}$, $\left[g_{1}, g_{2}\right] \notin \operatorname{span}\left\{g_{2}, \operatorname{ad}_{f} g_{2}\right\}$, for all $x \in \mathbb{R}^{4}$. Therefore, one concludes that the present example cannot be transformed into the flat triangular canonical form considered in [5] after a change of coordinates.

Example 2. As a practical engineering example, consider the reduced order model (current-fed) of an induction motor with three states

$$
\begin{aligned}
\dot{\omega} & =\frac{n_{p} M}{J L}\left(\psi_{a} i_{b}-\psi_{b} i_{a}\right)-\frac{T_{L}}{J}, \\
\dot{\psi}_{a} & =-\frac{R}{L} \psi_{a}-n_{p} \omega \psi_{b}+M \frac{R}{L} i_{a}, \\
\dot{\psi}_{b} & =-\frac{R}{L} \psi_{b}+n_{p} \omega \psi_{a}+M \frac{R}{L} i_{b},
\end{aligned}
$$

where $x=\left(x_{1}, x_{2}, x_{3}\right)=\left(\omega, \psi_{a}, \psi_{b}\right) \in \mathbb{R}^{3}$ is the state, $u=\left(u_{1}, u_{2}\right)=\left(i_{a}, i_{b}\right) \in$ $\mathbb{R}^{2}$ is the control, and $J, L, M, n_{p}, R, T_{L} \in \mathbb{R}$ are the (constant) parameters. See [15, p. 276] for details. This system has the form (1) and it meets the conditions of Theorem 3 at all $x \in \mathbb{R}^{3}$. In fact, $\operatorname{dim}\left(F_{k}\right)=\operatorname{dim}\left(G_{k}\right)=2+k$, for $0 \leq k \leq 1$ (see Remark 1). By Remark 3, in order to describe this system by the triangular form (3), it suffices to find a change of coordinates $z=\varphi(x)$ and a feedback $\beta(x)$ in which the corresponding control vector fields $g_{1}, g_{2}$ are described by the chained form (2) with $z=\varphi(x)$ and $u=\beta v$. In the previous academic example they were directly provided, but in this one they shall be constructed based on [14, Proposition 7]. This will allow the construction of a flat output accordingly.

Using the same notation as in [14, Proposition 7], it suffices to find smooth functions $h_{1}, h_{2}$ such that $L_{g_{1}} h_{1}=1, d h_{1} \Delta_{1}=d h_{2} \Delta_{2}=0$, where $\Delta_{1}=$ $\left\{g_{2},\left[g_{1}, g_{2}\right]\right\}$ and $\Delta_{2}=\left\{g_{2}\right\}$. An obvious choice is $h_{1}(x)=(M R)^{-1} L x_{2}$ and $h_{2}(x)=L^{-1} M R x_{1}-(J L)^{-1} n_{p} M x_{2} x_{3}$. Consequently, [14, Proposition 7] implies that $z_{1}=h_{2}(x), z_{2}=L_{g_{1}} h_{2}(x)=-2\left(J L^{2}\right)^{-1} n_{p} M R^{2} x_{3}, z_{3}=h_{1}(x)$ is the desired change of coordinates and that

$$
\beta=\left(\begin{array}{cc}
1 & 0 \\
L_{g_{1}}^{2} h_{1} & L_{g_{2}} L_{g_{1}} h_{2}
\end{array}\right)^{-1}=\left(\begin{array}{cc}
1 & 0 \\
0 & -\left(2 n_{p} M^{3} R^{2}\right)^{-1} J L^{3}
\end{array}\right)
$$

is the required (constant) feedback matrix. Such change of coordinates is in fact global, that is, it is a diffeomorphism from $\mathbb{R}^{3}$ onto $\mathbb{R}^{3}$, since 
$L_{g_{2}} L_{g_{1}} h_{2}(x) \neq 0$ for all $x \in \mathbb{R}^{3}$. One referes the reader to the proof of [14, Proposition 7] for details.

Now, following Remark 3, take

$$
\alpha=-\beta\left(\left\langle d z_{3}, f\right\rangle,\left\langle d z_{2}, f\right\rangle\right)=(M R)^{-1}\left(R x_{2}+n_{p} L x_{1} x_{3}, R x_{3}-n_{p} L x_{1} x_{2}\right) .
$$

After applying the global feedback $u=\alpha+\beta v$, one obtains that the closedloop system is described in the global coordinates $z=\left(z_{1}, z_{2}, z_{3}\right)=\varphi(x)$ by the triangular form (3):

$$
\dot{z}=\left(\phi_{1}(z), 0,0\right)+\left(z_{2}, 0,1\right) v_{1}+(0,1,0) v_{2},
$$

where

$\phi_{1}(z)=\frac{-2 J^{2} L^{6} z_{1} z_{2}^{2}-8 n_{p}^{2} M^{6} R^{4} z_{1} z_{3}^{2}+4 n_{p}^{2} M^{6} R^{4} z_{2} z_{3}^{3}+J^{2} L^{6} z_{2}^{3} z_{3}-8 L M^{5} R^{4} T_{L}}{8 J L^{2} M^{4} R^{3}}$.

According to the regularity conditions stated in the introduction, one concludes that $y=\left(z_{1}, z_{3}\right)$ is a flat output for the induction motor model above around the points that satisfy

$$
v_{1}+\partial \phi_{1} / \partial z_{2}(z)=v_{1}-\frac{J L^{4} z_{2}\left(4 z_{1}-3 z_{2} z_{3}\right)}{8 M^{4} R^{3}}+\frac{n_{p}^{2} M^{2} R z_{3}^{3}}{2 J L^{2}} \neq 0
$$

(in the $z$ coordinates), or equivalently, since $u_{1}=v_{1}$,

$$
u_{1}+\frac{n_{p} L\left(n_{p} x_{2}^{3}+2 J R x_{1} x_{3}+n_{p} x_{2} x_{3}^{2}\right)}{2 J M R^{2}} \neq 0
$$

(in the original coordinates). Note that $z=\varphi(x)$ and $u=\alpha+\beta v$, as well the flatness condition above, do not depend on the torque load $T_{L}$.

\section{Conclusion}

This work has established necessary and sufficient geometric conditions for (11) to be described by the triangular form (3), which is (essentially) flat. One has treated systems with only two inputs $(m=2)$. A generalization of the conditions here obtained to systems with $m>2$ inputs has been recently achieved in [16]. In terms of future research, the triangular form (3) may give rise to the development of constructive steering methods for systems with drift. The techniques described in [9] and [13] for the chained form (2) could point to that direction. 


\section{References}

[1] R. M. Murray, Nilpotent bases for a class of non-integrable distributions with applications to trajectory generation for nonholonomic systems, Math. Control Signals Systems 7 (1) (1995) 58-75.

[2] P. Martin, P. Rouchon, Feedback linearization and driftless systems, Math. Control Signals Systems 7 (1994) 235-254.

[3] S. Li, C. Xu, H. Su, J. Chu, Characterization and flatness of the extended chained system, in: Proc. of the 32nd Chinese Control Conference, Xi'an, China, 2013, pp. 1047-1051.

[4] H. B. Silveira, Formas triangulares para sistemas não-lineares com duas entradas e controle de sistemas sem arrasto em $\mathrm{SU}(n)$ com aplicações em mecânica quântica., Ph.D. thesis, University of São Paulo (USP), São Paulo, Brazil, In portuguese (February 2010).

[5] S. Bououden, D. Boutat, J.-P. Barbot, F. Kratz, A triangular canonical form for a class of 0-flat nonlinear systems, International Journal of Control 84 (2) (2011) 261-269.

[6] P. Martin, R. M. Murray, P. Rouchon, Flat systems, equivalence and trajectory generation, Tech. rep., available at http://cas.ensmp.fr/ rouchon (2003).

[7] J. Lévine, Analysis and Control of Nonlinear Systems: A Flatness-based Approach, Springer-Verlag, Berlin, 2009.

[8] H. Sira-Ramirez, S. K. Agrawal, Differentially Flat Systems, Marcel Dekker, New York, 2004.

[9] S. Sastry, Nonlinear Systems: Analysis, Stability and Control, SpringerVerlag, New York, 1999.

[10] W. Respondek, Transforming nonholonomic control systems into the canonical contact form, in: Proc. of the 40th IEEE Conf. on Decision and Control - CDC, Orlando, United States, 2001, pp. 1781-1786.

[11] K. Yang, Exterior Differential Systems and Equivalence Problems, Kluwer Academic Publishers, Dordrecht, 1992. 
[12] J. Dieudonné, Treatise on Analysis Vol. 4, Acad. Press, New York, 1974.

[13] D. Tilbury, R. M. Murray, S. R. Sastry, Trajectory generation for the n-trailer problem using Goursat normal form, IEEE Trans. Automat. Control 40 (5) (1995) 802-819.

[14] R. M. Murray, S. Sastry, Nonholonomic motion planning: Steering using sinusoids, IEEE Trans. Automat. Control 38 (5) (1993) 700-716.

[15] R. Marino, P. Tomei, Nonlinear Control Design: Geometric, Adaptive and Robust, Prentice Hall, London, 1995.

[16] F. Nicolau, Géométrie et platitude des systèmes de contrôle de poids différentiel minimal, Ph.D. thesis, L'Institut National des Sciences Appliquées (INSA), Rouen, France, In preparation (2014). 